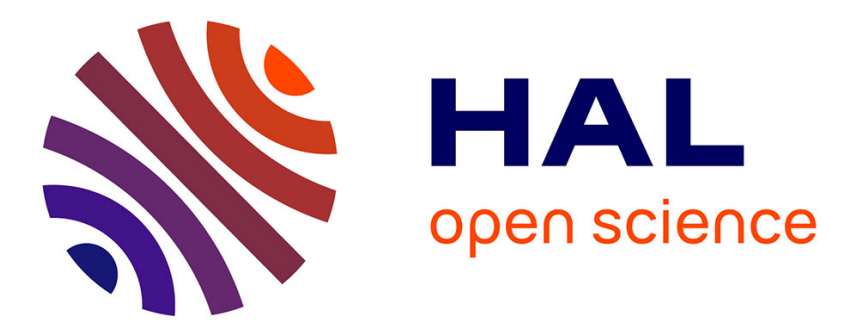

\title{
Influence d'additifs organiques à faible potentiel d'ionisation sur le fonctionnement des décharges autonomes dans les mélanges $\mathrm{CO} 2-\mathrm{N} 2-\mathrm{He}$ à la pression atmosphérique
}

R. Fabbro, J. Bruneteau, E. Fabre

\section{To cite this version:}

R. Fabbro, J. Bruneteau, E. Fabre. Influence d'additifs organiques à faible potentiel d'ionisation sur le fonctionnement des décharges autonomes dans les mélanges $\mathrm{CO} 2-\mathrm{N} 2-\mathrm{He}$ à la pression atmosphérique. Journal de Physique, 1977, 38 (9), pp.1071-1076. 10.1051/jphys:019770038090107100 . jpa-00208673

\section{HAL Id: jpa-00208673 https://hal.science/jpa-00208673}

Submitted on 1 Jan 1977

HAL is a multi-disciplinary open access archive for the deposit and dissemination of scientific research documents, whether they are published or not. The documents may come from teaching and research institutions in France or abroad, or from public or private research centers.
L'archive ouverte pluridisciplinaire HAL, est destinée au dépôt et à la diffusion de documents scientifiques de niveau recherche, publiés ou non, émanant des établissements d'enseignement et de recherche français ou étrangers, des laboratoires publics ou privés. 


\title{
INFLUENCE D'ADDITIFS ORGANIQUES \\ A FAIBLE POTENTIEL D'IONISATION \\ SUR LE FONCTIONNEMENT DES DÉCHARGES AUTONOMES \\ DANS LES MÉLANGES $\mathrm{CO}_{2}-\mathrm{N}_{2}-\mathrm{He}$ \\ A LA PRESSION ATMOSPHÉRIQUE $(*)(* *)$
}

\author{
R. FABBRO, J. BRUNETEAU et E. FABRE
}

Laboratoire de Physique des Milieux Ionisés (***), Ecole Polytechnique, 91128 Palaiseau Cedex, France

(Reçu le 22 décembre 1976, révisé le 27 avril 1977, accepté le 12 mai 1977)

\begin{abstract}
Résumé. - L'adjonction de molécules organiques à faible potentiel d'ionisation aux mélanges laser $\mathrm{CO}_{2}-\mathrm{N}_{2}-\mathrm{He}$ a pour effet de modifier les mécanismes d'ionisation dans le milieu. En plus de l'ionisation collisionnelle de l'additif, l'ionisation de l'additif par effet Penning à l'aide des métastables de l'azote permet de diminuer fortement le champ d'entretien des décharges autoentretenues. Ceci a pour conséquence d'augmenter à la fois le rendement et la stabilité de ces décharges.
\end{abstract}

\begin{abstract}
The introduction of low ionization potential organic molecules to $\mathrm{CO}_{2}-\mathrm{N}_{2}-\mathrm{He}$ laser mixtures modifies the ionization mechanisms of the gas. Added to the collisional ionization of the seed gas, the Penning ionization of the additive by metastable $\mathrm{N}_{2}$ strongly reduces the characteristic quasisteady electric field of the self-sustained glow discharges. There is a consequent increase in efficiency and in stability of these discharges.
\end{abstract}

1. Introduction. - L'utilisation de la photoionisation par U.V. pour la préionisation de mélanges laser gazeux à la pression atmosphérique est une méthode très efficace pour initier dans des volumes importants des décharges diffuses homogènes [1-3]. Une amélioration de cette technique [4] consiste à introduire dans le mélange gazeux des additifs organiques (xylène, tri-n-propylamine) à faible potentiel d'ionisation, donc plus facilement photoionisables par les U.V. Cette technique est donc devenue assez utilisée ; si l'on excepte les travaux de Reits et Olbertz [5, 6], il s'avère que l'influence exacte de ces additifs sur le déroulement de la décharge est très mal connue. Nous présentons dans cette étude les résultats préliminaires concernant d'une part les modifications apportées par l'introduction de ces additifs dans les mécanismes d'ionisation existant dans les décharges autoentretenues $(\S 3)$ et d'autre part les conséquences qui en résultent, à savoir : diminution de la tension d'entretien de la décharge,

(*) Etude réalisée avec le concours de la Direction des Recherches et Moyens d'Essais.

$\left.{ }^{* *}\right)$ Communication présentée au Congrès National de Physique des Plasmas, Paris, 6-10 décembre 1976.

(***) Groupe de Recherche du C.N.R.S. augmentation de l'énergie déposée et amélioration de la stabilité de la décharge $(§ 4)$.

2. Dispositif expérimental. - Le dispositif expérimental utilisé est représenté à la figure $1 a$. La décharge s'effectue entre deux électrodes profilées en cuivre de $8 \times 8 \mathrm{~cm}^{2}$ de surface. La distance interélectrode peut varier jusqu'à $3 \mathrm{~cm}$. Le circuit de décharge comprend une capacité de décharge, couramment de $6,6 \mathrm{nF}$, une inductance variable, une résistance très faible $(1 \Omega)$ permettant la mesure du courant, ainsi qu'un éclateur dont le moment de mise à feu est réglable par rapport à la préionisation. La tension aux bornes de la décharge est mesurée à l'aide d'une sonde TEKTR. P 6015. Il est possible d'introduire dans l'enceinte de décharge contenant le mélange gazeux $\mathrm{CO}_{2}-\mathrm{N}_{2}-\mathrm{He}$ à la pression atmosphérique une quantité connue d'additif pouvant varier de 1 à 4000 ppm (parties par million). Le gaz circulant en permanence, avec un débit d'environ $10 \mathrm{l} / \mathrm{mn}$.

Les photons U.V. servant à la préionisation sont émis par un arc produit par la décharge d'un condensateur de $50 \mathrm{nF}$ chargé sous $30 \mathrm{kV}$. L'enceinte où s'effectue cet arc contient de l'azote pur $(p=1 \mathrm{~atm})$ et se trouve séparée de l'enceinte de décharge par un 


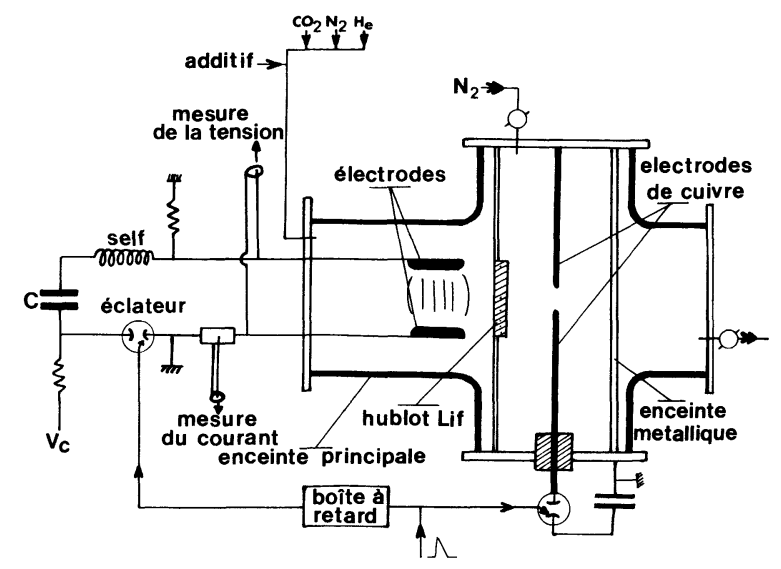

(a)

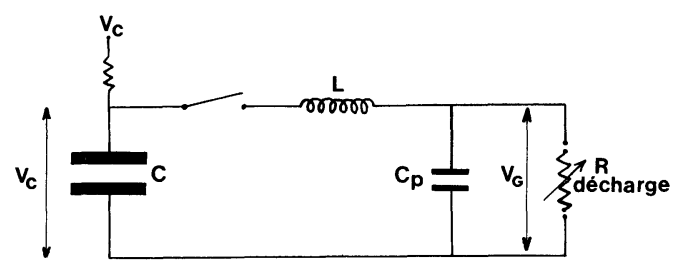

(b)

FIG. 1. - a) Schéma du dispositif expérimental. b) Circuit électrique équivalent.

[a) Schema of the experimental device. b) Equivalent electrical circuit.]

hublot de LiF. Ceci permet d'obtenir un spectre d'U.V. indépendant du mélange étudié.

Une étude précédente [7] nous avait permis d'étudier la densité électronique résultant de la préionisation par U.V. Nous avions constaté que pour un spectre d'U.V. donné, la densité électronique maximale obtenue ne dépendait que de la concentration d'additif et de $\mathrm{CO}_{2}$ dans le mélange utilisé. Compte tenu des caractéristiques de notre montage expérimental, on peut exprimer la valeur de la densité électronique au moment de la mise à feu de l'éclateur (environ $2 \mu \mathrm{s}$ après le début de la préionisation), par la relation suivante :

$$
\begin{array}{r}
n_{\mathrm{e} 0}\left(\mathrm{~cm}^{-3}\right) \approx 2,5 \times 10^{9} \cdot c \cdot \exp \left(-1,2 \times 10^{-2} \cdot c\right) \times \\
\times \exp (-0,47 \cdot r)
\end{array}
$$

$c$ étant la concentration en tri-n-propylamine exprimée en ppm et $r$ étant le pourcentage en $\mathrm{CO}_{2}$ du mélange. Ainsi, poưr un mélange contenant $10 \%$ de $\mathrm{CO}_{2}$ et $50 \mathrm{ppm}$ d'additif, la densité de préionisation est de $6 \times 10^{8} \mathrm{~cm}^{-3}$. D'autre part, le centre de la décharge se trouvant à $18 \mathrm{~cm}$ de l'arc, on peut estimer à environ $30 \%$ la variation de $n_{\mathrm{e} 0}$ d'une extrémité à l'autre des électrodes; du fait de la dissymétrie de l'illumination en U.V.

3. Etude de la décharge diffuse. - 3.1 Résultats EXPÉRIMENTAUX. - L'interprétation des caractéris- tiques courant-tension des décharges autoentretenues pour l'étude des processus d'ionisation ou de pertes par attachement et par recombinaison nécessite l'utilisation de modèles de décharges. Nous utiliserons tout d'abord l'analyse de Judd et Wada [8].

Denes et Lowke [9] ont montré que pour un mélange $\mathrm{CO}_{2}-\mathrm{N}_{2}-\mathrm{He}$ donné, le rapport $E / N$ ( $E$ champ électrique, $N$ densité de neutre), lorsque le courant est stationnaire, se stabilise à une valeur $E_{\mathrm{G}} / N$ qui est indépendante de la densité de courant de décharge, de la pression totale et de la distance interélectrode. $E_{\mathrm{G}} / N$ est tel que : $\alpha\left(E_{\mathrm{G}} / N\right)=a\left(E_{\mathrm{G}} / N\right), \alpha$ et $a$ étant respectivement les coefficients d'ionisation collisionnelle et d'attachement dissociatif.

A l'aide du circuit de décharge (Fig. 1b), où l'on néglige momentanément la présence de la capacité parasite $C_{\mathrm{p}}$ qui se révèle très importante pour la période transitoire de formation de la décharge, nous obtenons alors aisément l'évolution temporelle du courant de décharge :

$$
I(t)=\sqrt{\frac{C}{L}}\left(V_{\mathrm{C}}-V_{\mathrm{G}}\right) \sin (\omega \cdot t) .
$$

Avec $\omega^{-1}=\sqrt{L C}, V_{\mathrm{C}}$ étant la tension de charge de $C$ et $V_{\mathrm{G}}=E_{\mathrm{G}} . d$ la tension supposée constante aux bornes de la décharge de distance interélectrode $d$. Pour $t=\frac{\pi}{2} \sqrt{L C}$, le courant est maximum et vaut :

$$
I_{\max }=\sqrt{\frac{C}{L}}\left(V_{\mathrm{C}}-V_{\mathrm{G}}\right) .
$$

Nous avons représenté à la figure 2 la variation de $I_{\max }$ en fonction de $V_{\mathrm{C}}$ pour les mélanges $\mathrm{CO}_{2}-\mathrm{N}_{2}-\mathrm{He}$ : $1-1-8,1-2-3,1-7-0$ contenant des concentrations variables d'additif. La pente commune de ces droites ne dépend que de la capacité et de la self du circuit ;

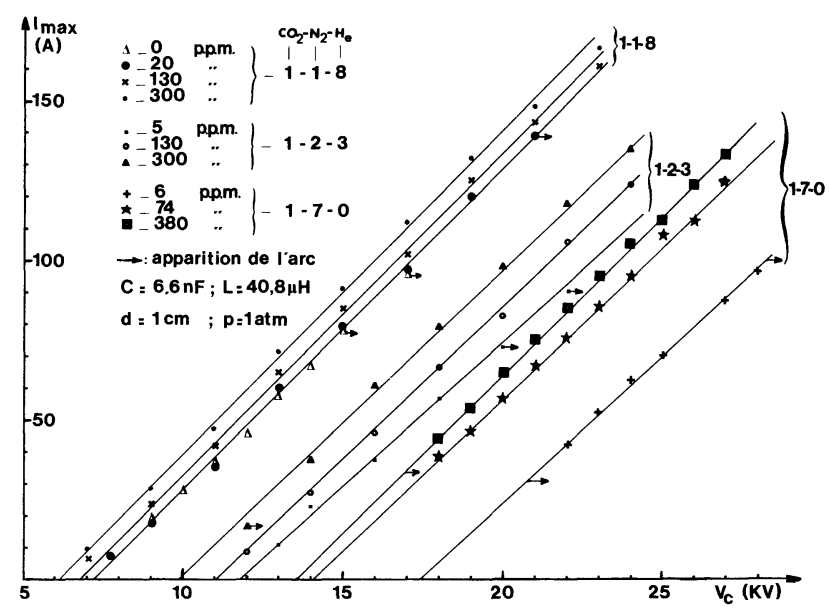

Fig. 2. - Courant maximal de décharge en fonction de la tension de charge du condensateur. Etude de différents mélanges $\mathrm{CO}_{2}-\mathrm{N}_{2}-\mathrm{He}+$ Additif.

[Maximum discharge current $v s$. the initial voltage of the capacitor for several $\mathrm{CO}_{2}-\mathrm{N}_{2}-\mathrm{He}+$ Additives mixtures.] 


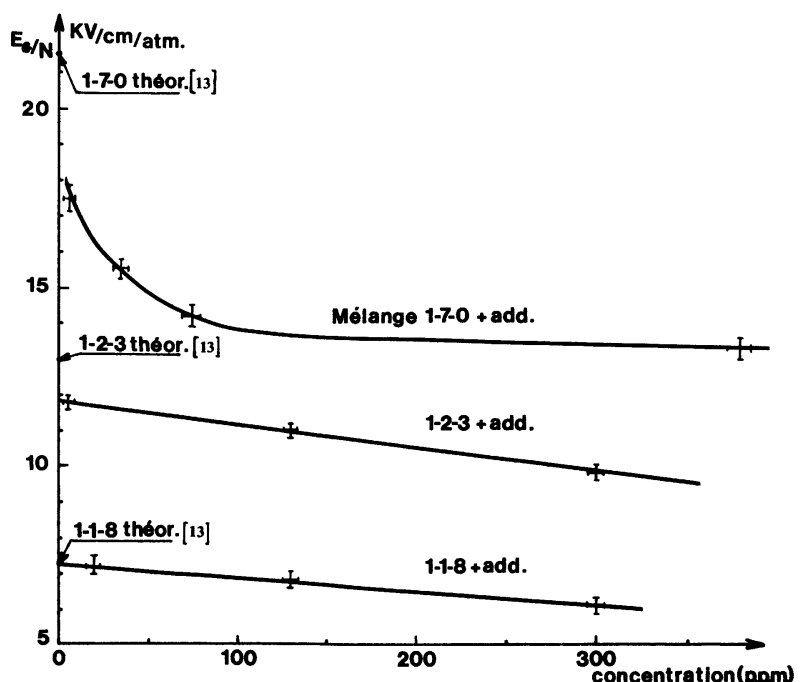

FIG. 3. - Champ d'entretien de la décharge en fonction de la concentration de tri-n-propylamine pour différents mélanges $\mathrm{CO}_{2}-\mathrm{N}_{2}-\mathrm{He}$.

[Quasisteady electric field of the self-sustained glow discharge $v s$. the concentration of tri-n-propylamine for several $\mathrm{CO}_{2}-\mathrm{N}_{2}-\mathrm{He}$ mixtures.] (a)
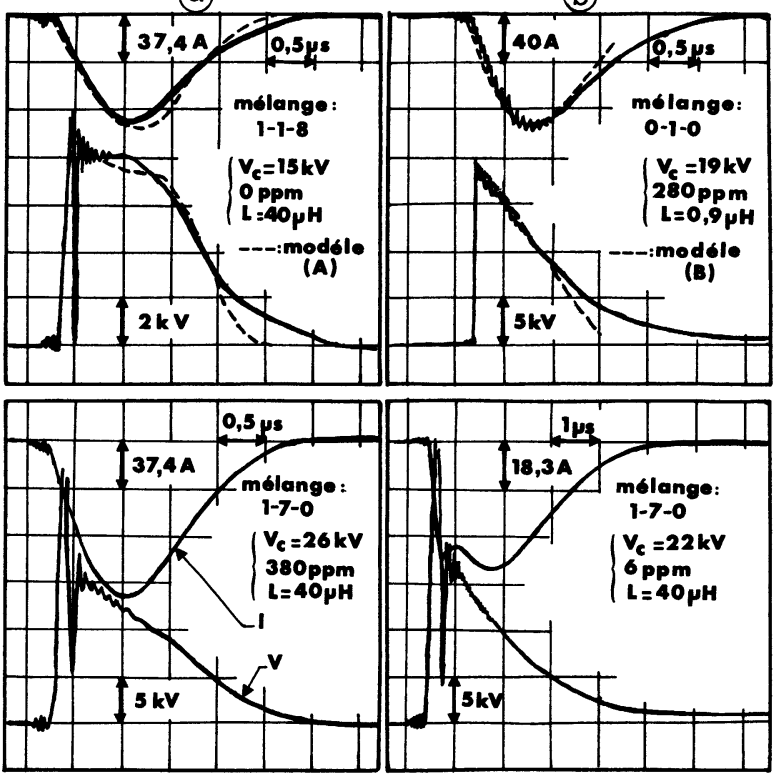

(C)
Fig. 4. - Evolution temporelle de la tension et du courant pour des décharges autoentretenues dans différents mélanges $\mathrm{CO}_{2}-\mathrm{N}_{2}-\mathrm{He}+$ Additif.

[Temporal characteristics of self-sustained discharges for several $\mathrm{CO}_{2}-\mathrm{N}_{2}-\mathrm{He}+$ Additives mixtures.]

elle est en bon accord avec la valeur théorique prévue par la relation (3). Nous vérifions également que $I=I_{\max }$ pour $t=0,8 \mu \mathrm{s}$ (Fig. 4).

Cette méthode nous permet donc de déterminer la valeur de $E_{\mathrm{G}} / N$ de chaque mélange. Les résultats de la figure 2 nous montrent d'une part que, pour un mélange donné, $E_{\mathrm{G}} / N$ est une fonction décroissante de la concentration d'additifs présente et que d'autre part, cet effet est d'autant plus sensible que le mélange $\mathrm{CO}_{2}-\mathrm{N}_{2}-\mathrm{He}$ pur avait un $E_{\mathrm{G}} / N$ élevé (voir Fig. 3).

\subsection{INTERPRÉTATION DES RÉSULTATS. -} 3.2.1 L'observation de la tension aux bornes de la décharge montre que celle-ci n'est pas constante au cours du temps. Ceci est lié au fait que la décharge n'est pas stationnaire : au départ, lors de la montée de courant, $\mathrm{d} n_{\mathrm{e}} / \mathrm{d} t>0$ entraîne $\alpha>a$, soit $E / N>E_{\mathrm{G}} / N$, où $E_{\mathrm{G}} / N$ est le champ d'entretien d'un courant stationnaire $(\alpha=a)$. En fait $\alpha(E)$ étant une fonction à variation très rapide, $E$ reste voisin de $E_{\mathrm{G}}$, mais par excès ; ceci explique la possibilité d'appliquer le modèle de Judd et Wada à notre cas, la tension variant peu pendant la montée du courant qui dure $\frac{\pi}{2} \sqrt{L C}$. Par contre au cours de la décroissance de courant, on observe des variations importantes de la tension, qui sont à relier aux variations plus lentes de la fonction $a(E)$.

Une représentation plus précise de l'évolution temporelle de la tension et du courant est obtenue à l'aide du modèle simplifié de Kline et Denes [11] où on écrit (modèle A) :

$$
\begin{aligned}
\frac{\mathrm{d} n_{\mathrm{e}}}{\mathrm{d} t} & =(\alpha-a) n_{\mathrm{e}} v_{\mathrm{e}} \\
i & =n_{\mathrm{e}} v_{\mathrm{e}} e S \\
V(t) & =V(0)-L \frac{\mathrm{d} i}{\mathrm{~d} t}-\frac{1}{C} \int_{0}^{t} i \mathrm{~d} t .
\end{aligned}
$$

On ne tient donc compte que des effets collisionnels pour l'évolution de la densité électronique. Par ailleurs la recombinaison électronique est négligeable.

La figure $4 a$ montre l'application de ce modèle au cas du mélange 1-1-8, sans additif. Les valeurs de $\alpha$, $a$ et $v_{e}$ sont celles calculées en fonction du champ électrique par Lowke, Phelps, Irwin [13] pour le même mélange. L'évolution de la tension, avec ou sans $\mathrm{N}_{2}$, est assez semblable, à ceci près que l'introduction de tri-N-propylamine a pour effet de supprimer le plateau de tension lors de la montée du courant. On n'observe pas une décroissance exponentielle du courant comme celle observée par Reitz et Olbertz [5], ceci étant lié aux différences de circuit de décharge.

3.2.2 Les résultats des figures 2 et 3 montrent que la présence d'additif introduit dans le mélange gazeux un processus d'ionisation qui a pour effet d'augmenter la densité électronique et qui par conséquent entraîne une réduction du champ d'entretien de la décharge. Deux processus peuvent être à l'origine de cette ionisation supplémentaire.

Lobanov et Suchov [10] ont montré la possibilité d'une ionisation collisionnelle par les électrons de l'additif. Nous pensons que cet effet est négligeable ; en effet compte tenu, d'une part, du faible potentiel d'ionisation $(7,23 \mathrm{eV})$ de la tri-N-pr. et de la forme de la fonction de distribution des énergies élec- 
troniques [13] et, d'autre part, de la faible concentration d'additif utilisée dans nos expériences, il faudrait une section efficace d'ionisation par les électrons très élevée pour que le coefficient d'ionisation soit important. Ce n'est pas le cas, car en ajoutant une concentration très élevée de tri- $\mathrm{N}$-propylamine $(4000 \mathrm{ppm})$ à des mélanges $\mathrm{CO}_{2}-\mathrm{He}$, nous n'avons observé qu'une très faible variation de $E_{\mathrm{G}} / N$. Ainsi pour des mélanges (1-0-9) et (1-0-3) $E_{\mathrm{G}} / N$ varie respectivement de 5,4 à $4,8 \mathrm{kV} / \mathrm{cm} / \mathrm{atm}$ et de 8 à $7,4 \mathrm{kV} / \mathrm{cm} / \mathrm{atm}$. Par contre, des concentrations d'additif bien plus faibles $(<400 \mathrm{ppm})$ produisent des variations de $E_{\mathrm{G}} / N$ beaucoup plus importantes dans le cas de mélanges contenant de l'azote.

3.2.3 Ces dernières observations vont dans le sens de l'existence d'un mécanisme d'ionisation lié à la fois à la présence de l'additif et de l'azote. L'hypothèse de Reits et Olbertz $[5,6]$ d'une ionisation par effet Penning de l'additif est donc confirmée par nos expériences. La création de métastables d'azote $\left(\mathrm{a}^{1} \pi_{\mathrm{g}}, \mathrm{a}^{\prime}{ }^{1} \Sigma_{\mathrm{u}}^{-}\right.$) lors de la phase transitoire de formation de 'la décharge permet d'expliquer nos observations. C'est dans un mélange azote-tri-N-propylamine qu'apparaît le plus clairement le rôle des métastables. Avec une self minimisée de $0,9 \mu \mathrm{H}$, l'évolution du courant n'est plus contrôlée par les impédances externes du circuit (le terme $L \frac{\mathrm{d} i}{\mathrm{~d} t}$ est négligeable), mais à l'intérieur même de la décharge par les variations de la densité électronique et du champ électrique. Si $N_{0}^{*}$ est la densité de métastables, créés au cours de la formation de la décharge, on peut adopter le modèle suivant (modèle B) :

$$
\frac{\mathrm{d} n_{\mathrm{e}}}{\mathrm{d} t}=-a n_{\mathrm{e}}+K N_{\mathrm{add}} N_{0}^{*} \mathrm{e}^{-K N_{\mathrm{add}} t}
$$

la recombinaison électronique étant encore négligeable

et

$$
i=e S n_{\mathrm{e}} v_{\mathrm{e}}(V)
$$

$$
\frac{\mathrm{d} V}{\mathrm{~d} t}=-\frac{i}{C}
$$

On admet donc que la durée de vie des métastables dans le mélange azote + additif est liée à la désexcitation Penning. On obtient un accord raisonnable avec les expériences (Fig. $4 b$ et 5) pour $N_{0}^{*}=10^{14} \mathrm{~cm}^{-3}$, $a=10^{5} \mathrm{~s}^{-1}, K=3,7 \times 10^{-12} \mathrm{~cm}^{3} \cdot \mathrm{s}^{-1}$. La densité initiale de métastables identique à celle rapportée par Reitz et Olbertz [6] représente environ 1/100 de l'énergie initialement stockée dans la capacité. Dans l'azote, l'attachement s'effectue sur des impuretés électronégatives; on sait que, en l'absence d'additif, $E / N=28,9 \mathrm{kV} / \mathrm{cm} / \mathrm{atm}$ [12]. Dans notre cas, on observe qu'en présence de $280 \mathrm{ppm}$ de tri-N-propylamine, le champ $E / N$ est de $11,5 \mathrm{kV} / \mathrm{cm} / \mathrm{atm}$; pour un tel champ l'attachement électronique est donc plus important que l'ionisation collisionnelle de l'azote.

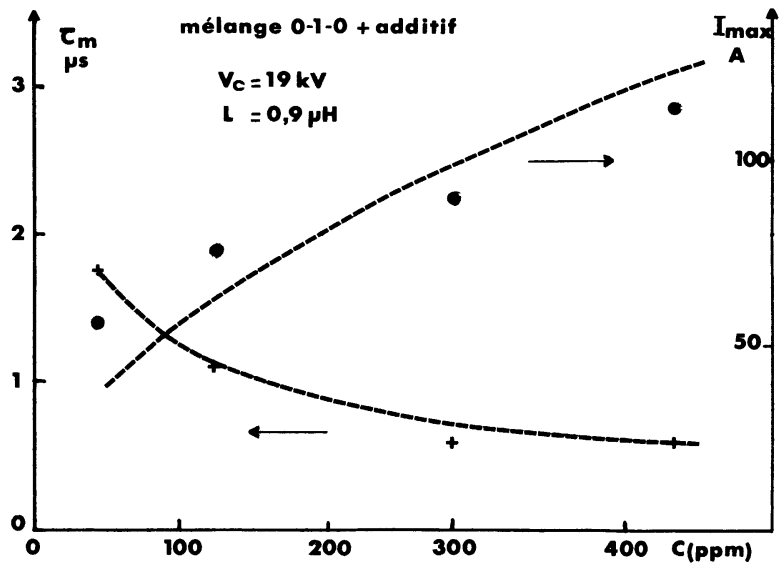

FIG. 5. - (+) Retard avec lequel apparaît le maximum de courant et $(\bullet)$ Amplitude de ce maximum en fonction de la concentration d'additif dans $\mathrm{N}_{2}(\bullet,+$ points expérimentaux, courbes - - - calculées par le modèle B).

$[(+)$ Delay time of the current maximum and $(\bullet)$ value of this current maximum vs. the additive concentration in $\mathrm{N}_{2}(\bullet,+$ experimental points, curves - - - computed by model B).]

La valeur de $a$ utilisée $\left(10^{5} \mathrm{~s}^{-1}\right)$ recoupe des mesures d'attachement électronique dans un plasma d'azote généré par photoionisation [7]

3.2.4 La présence de gaz carbonique a pour effet une désexcitation des métastables avec un taux de réaction de $1,2 \times 10^{-13} \mathrm{~cm}^{3} \mathrm{~mole}^{-1} \mathrm{~s}^{-1}$ [6]. Ceci donne une explication à l'effet moins important joué par l'additif dans la décharge en présence de $\mathrm{CO}_{2}$ (Fig. 3). Une description de la décharge devrait alors reprendre les éléments du modèle de Kline et Denes en y incluant l'effet des métastables : la figure $4 d$ montre un cas où apparaissent d'abord un maximum de courant dû aux effets collisionnels, puis un autre retardé de $1,3 \mu$ s dû à l'effet Penning. Avec une concentration d'additif plus forte les deux maxima sont confondus, à un instant dépendant de la self du circuit comme dans le modèle de Judd et Wada.

4. Conséquences de la diminution de $E_{\mathrm{G}} / N$ sur le rendement et la stabilité des décharges autoentretenues.

- 4.1 Amélioration DU RENDEMENT. - Nous savons que pour un mélange $\mathrm{CO}_{2}-\mathrm{N}_{2}-\mathrm{He}$ donné, il existe une valeur de $E / N$ optimisant le transfert de l'énergie électrique de la décharge sur les niveaux vibrationnels du $\mathrm{CO}_{2}$ et $\mathrm{N}_{2}$ intéressant l'effet laser. Dans les décharges autoentretenues, cette valeur est bien inférieure à $E_{\mathrm{G}} / N$. La présence d'additif ayant pour effet de diminuer ce champ de fonctionnement, il est donc possible par cette méthode d'améliorer le rendement des décharges autoentretenues. Nous avons vu que l'effet des additifs est d'autant plus sensible que le champ $E_{\mathrm{G}} / N$ initial était élevé ; cette solution intéressera donc davantage les mélanges $\mathrm{CO}_{2}-\mathrm{N}_{2}-\mathrm{He}$ à faible concentration d'hélium qui en outre présentent la particularité d'avoir un rendement théorique plus important $[13,14]$. 
Ainsi par exemple, pour le mélange $\mathrm{CO}_{2}-\mathrm{N}_{2}-\mathrm{He}$ : $1-7-0, E_{\mathrm{G}} / N=21 \mathrm{kV} / \mathrm{cm} / \mathrm{atm}$ [9]. En ajoutant $380 \mathrm{ppm}$ de Tri-n-propylamine, nous abaissons $E_{\mathrm{G}} / N$ à $13 \mathrm{kV} / \mathrm{cm} / \mathrm{atm}$. Cette valeur est alors très proche du champ optimum pour le rendement maximum qui est de $11 \mathrm{kV} / \mathrm{cm} / \mathrm{atm}$ [13]. Cet effet explique donc les rendements élevés obtenus dans le cas des décharges autoentretenues par Reits et Olbertz [6].

4.2 Stabilisation De LA DÉCHARGE. - La stabilité des décharges est un problème très complexe qui dépend de nombreux paramètres dont le rôle est actuellement peu connu $[15,16]$. Nous présentons ici quelques résultats expérimentaux concernant les modifications apportées par l'introduction de ces additifs sur la stabilité des décharges.

Nous observons tout d'abord que la concentration en $\mathrm{CO}_{2}$ du mélange étudié est un paramètre important pour la stabilité de la décharge. Ainsi pour nos conditions expérimentales à l'aide d'un circuit possédant un temps $\tau$ de décharge donné $(\tau=\pi \sqrt{L C}=1,63 \mu \mathrm{s}$ par exemple pour $C=6,6 \mathrm{nF}$ et $L=40 \mu \mathrm{H}$ ), nous constatons qu'il est impossible d'obtenir, sans l'adjonction d'additifs, une décharge homogène dans des mélanges contenant environ plus de $10 \%$ de $\mathrm{CO}_{2}$; la concentration d'additif à ajouter étant alors une fonction croissante de la concentration en $\mathrm{CO}_{2}$. Bien que le $\mathrm{CO}_{2}$ ait pour effet de diminuer $n_{\mathrm{e} 0}$ (éq. (1)), nous pensons que cette réduction de $n_{\mathrm{e} 0}$ ne joue pas un rôle dominant pour la stabilité de la décharge.

En effet, la densité initiale de préionisation semble avoir peu d'influence sur la stabilité de la décharge. Ainsi, par exemple, pour le mélange 1-2-3, avec les caractéristiques précédentes du circuit, nous avons modifié $n_{\mathrm{e} 0}$ dans une très large gamme par différentes méthodes : soit en retardant le moment d'application de la tension de décharge de $0,2 \mu \mathrm{s}$ à $2 \mathrm{~ms}$ par rapport au début de la préionisation, soit en diminuant le flux d'U.V. de préionisation, ou encore en augmentant très fortement la concentration en additif (éq. (1)). Dans tous les cas, malgré l'importante réduction de $n_{\mathrm{e} 0}$ (de plus de 4 ordres de grandeur) la décharge est homogène ; alors qu'il n'en est pas de même en absence d'additif où nous estimons que $n_{\mathrm{e} 0}<10^{7}$ électrons $/ \mathrm{cm}^{3}$. Il apparaît donc qu'à partir d'un certain degré minimum de préionisation [17], c'est avant tout la présence de l'additif qui conditionne la stabilité de la décharge.

L'apparition de l'arc dépend aussi d'un autre paramètre : la tension de décharge $V_{\mathrm{C}}$ du condensateur. Pour un circuit et un mélange $\mathrm{CO}_{2}-\mathrm{N}_{2}-\mathrm{He}+$ additifs donnés, nous constatons qu'il existe 2 régions de $V_{\mathrm{C}}$ pour lesquelles la décharge est instable (voir Fig. 2); il semblerait qu'il soit possible de corréler l'apparition de l'arc avec la durée de la phase transitoire de formation de la décharge. En effet, pendant cette phase, la tension aux bornes de la décharge peut atteindre pratiquement $2 V_{\mathrm{C}}$, à cause de la présence d'une capacité parasite $C_{\mathrm{p}}$ (voir Fig. $1 b$ ) aux bornes de la décharge [18]. Dans notre montage, nous estimons que $C=30 \mathrm{pF}$. C'est cette surtension qui est responsable de l'augmentation de $n_{\mathrm{e}}$ qui passe de $n_{\mathrm{e} 0}$ (densité de préionisation) à environ $10^{12}-10^{13}$ électrons $/ \mathrm{cm}^{3}$, valeur de fonctionnement [11]; la durée de cette période transitoire étant de l'ordre de $\pi \sqrt{\left(L C_{\mathrm{p}}\right)}$ (environ $100 \mathrm{~ns}$ dans notre cas). Nous expliquons ainsi que lorsque $V_{\mathrm{C}}$ est élevé, la surtension appliquée pendant un temps trop long conduit à une instabilité d'ionisation collisionnelle [19]. Par contre, lorsque $V_{\mathrm{C}}$ est faible, il semblerait que la surtension soit insuffisante pour que la décharge s'amorce d'une façon homogène. Ces différentes observations suggèrent l'existence d'une relation entre la surtension et la durée de celle-ci pour réaliser un conditionnement optimal de la décharge.

Ces expériences préliminaires sembleraient montrer que dans le cas où l'effet Penning est le processus d'ionisation dominant, celle-ci fonctionne alors d'une façon analogue aux décharges entretenues par des agents extérieurs (faisceaux d'électrons). Nous savons alors que dans ce cas, les décharges sont beaucoup plus stables [20].

5. Conclusion. - Nous avons vu que l'introduction de molécules à faible potentiel d'ionisation au mélange $\mathrm{CO}_{2}-\mathrm{N}_{2}-\mathrm{He}$ a pour effet d'augmenter l'ionisation du milieu et par conséquent de diminuer le champ d'entretien $E_{\mathrm{G}} / N$ de la décharge. Les conséquences de cet effet sont très importantes : il en résulte d'une part une amélioration du rendement pour les lasers utilisant des décharges autoentretenues et d'autre part une augmentation importante de la stabilité des décharges. Des expériences ultérieures devraient permettre d'une part d'améliorer la connaissance des mécanismes de production et de désexcitation des métastables (effet de la densité électronique, de l'hélium, du $\mathrm{CO}_{2}$ et de l'additif) et d'autre part de déterminer les additifs présentant les meilleures caractéristiques (faible potentiel d'ionisation, sections efficaces d'ionisation collisionnelle élevée).

\section{Bibliographie}

[1] Seguin, H. J., Tulip, J., Appl. Phys. Lett. 21 (1972) 414.

[2] Judd, O. P., WadA, J. Y., Appl. Phys. Lett. 22 (1973) 95.

[3] Richardson, M. C., Alcock, A. J., Leopold, K., Burtyn, P., Proceedings of the Symposium on High Power Molecular Laser (Quebec) 1972.

[4] JAVAN, A., LEVINE, J. S., I.E.E.E. J. Quantum Electron. 8 (1972) 827.
[5] Reits, B. J., Olbertz, A. H. M., Appl. Phys. Lett. 27 (1975) 24.

[6] Olbertz, A. H. M., ReIts, B. J., Appl. Phys. Lett. 28 (1976) 199.

[7] Fabbro, R., Bruneteau, J., Fabre, E., J. Physique Colloq. 36 (1975) C6-25.

[8] JudD, O. P., WADA, J. Y., I.E.E.E. J. Quantum Electron. 10 (1974) 12.

[9] Denes, L. J., Lowke, J. J., Appl. Phys. Lett. 23 (1973) 130. 
[10] Lobanov, A. N., Suchkov, A. F., Sov. J. Quant. Electron. 4 (1975) 843.

[11] Kuine, L. E., Denes, L. J., J. Appl. Phys. 46 (1975) 1567.

[12] Meyer, J., Lee, C. S., J. Phys. D 4 (1971) 168.

[13] Lowke, J. J., Phelps, A. V., Irwin, B. W., J. Appl. Phys. 44 (1973) 4664

[14] Taylor, L. H., Weaver, L. A., Lieberman, R. W., Bull. Am. Phys. Soc. 20 (1975) 231.
[15] HaAs, R. A., Phys. Rev. A 8 (1973) 1017.

[16] JACOB, J. H., Mani, S. A., Appl. Phys. Lett. 26 (1975) 53.

[17] Palmer, A. J., Appl. Phys. Lett. 25 (1974) 138.

[18] Bushnell, A. H., Gundersen, M., BurKes, J. R., I.E.E.E. J. Quantum Electron. 12 (1976) 447.

[19] Dyer, P. E., James, D. J., J. Appl. Phys. 46 (1975) 1679.

[20] Nigham, W. L., Invited Paper 11th International Conference on Phenomena in Ionized Gases (Prague) 1973. 